\title{
Granulocyte colony-stimulating factor receptor mutations in severe chronic neutropenia and acute myeloid leukaemia: biological and clinical significance
}

\author{
IVO P. TOUW PhD \\ Staff Scientist \\ Department of Haematology, The Dr Daniel den Hoed Cancer Centre and Institute of Haematology, \\ Room Ee 1330c, Erasmus University. Dr Molewaterplein 50, 3000 DR Rotterdam, The Netherlands
}

\begin{abstract}
Blood cell formation is governed by the haemopoietic growth factors that control the proliferation, maturation and survival of the haemopoietic progenitor cells via activation of receptors expressed on the cell membrane. Most of these receptors share structural features and have been grouped in the haemopoietin or class I receptor superfamily. Recently considerable progress has been made in elucidating the regions critical for the function of these receptors and the signal transduction mechanisms that they activate. Moreover, it has become clear that certain clinical haematological conditions can be linked to specific defects in these receptors. The significance of defects in the receptor for granulocyte colony-stimulating factor (G-CSF) in the pathogenesis of severe congenital neutropenia and acute myeloid leukaemias is discussed.
\end{abstract}

Key words: haemopoietic growth factor receptors; signal transduction; severe congenital neutropenia; acute myeloid leukaemia; G-CSF receptor defects.

Haemopoietic growth factors (HGFs) stimulate the proliferation and development of haemopoietic precursor cells via binding to specific cell surface receptors. The majority of HGF receptors (HGF-R) belong to the superfamily of haemopoietin or cytokine receptors, also referred to as the class I receptor superfamily (Bazan, 1990; Cosman, 1993). Activation of these receptors involves the formation of dimeric or oligomeric complexes of receptor structures. Some HGF-R are composed of heteromeric complexes, comprising distinct receptor chains. For example, the receptors of interleukin (IL-) 2, IL-3, IL-5 and granulocyte-macrophage colonystimulating factor (GM-CSF) (Miyajima et al, 1993). Other receptors, for example those of granulocyte-CSF (G-CSF) and erythropoietin (EPO), are thought to form homodimeric complexes upon growth factor binding (Fukunaga et al, 1991; Cosman, 1993). 
Severe chronic neutropenia (SCN) and acute myeloid leukaemia (AML) are disease conditions characterized by a myeloid maturation arrest in the bone marrow. Because SCN patients have a highly increased probability of developing AML, it has been postulated that common or functionally overlapping mechanisms may be involved in these diseases. A number of research groups have recently paid attention to the possibility that abnormalities in responses to GM-CSF and G-CSF, two of the major HGFs implicated in the control of myeloid differentiation, might contribute to the pathogenesis of SCN and AML.

The major principles of signal transduction from the HGF-Rs will be discussed. Next, experimental and clinical evidence for the involvement of HGF-R abnormalities for the development of disorders of the haemopoietic system will be summarized. Finally, the biological and clinical consequences of signalling defects of the GCSF receptor in SCN and AML will be discussed.

\section{Structure and signalling function of class $I$ haemopoietin receptors}

Class I receptors are glycoproteins with an extracellular N-terminal domain, a single hydrophobic membrane spanning domain and a $\mathrm{C}$ terminal cytoplasmic domain. Characteristic of these receptors is the cytokine receptor homology region in the extracellular domain that is required for HGF binding. The intracellular domains of class I receptors show little overall homology. Structure/function analysis of the G-CSF receptor in appropriate cell line models showed that distinct functional subdomains in the cytoplasmic region of class I type cytokine receptors may be involved in transduction of proliferation and maturation signals (Dong et al, 1993; Fukunaga et al, 1993). More recently, similar findings have been reported for differentiation induced by the thrombopoietin (TPO) receptor (Porteu et al, 1996) and the IL-6 signal transducer GP130 (Yamanaka et al, 1996).

Although class I cytokine receptors activate cytoplasmic signalling substrates via tyrosine (Tyr)-phosphorylation, they do not possess Tyr kinase activity themselves. Instead, protein tyrosine phosphorylation of downstream substrates is achieved via activation of cytoplasmic protein Tyr kinases (PTKs). The Janus kinases (JAKs) comprise a PTK family with specific structural features that contains at least five members, four of which (JAKs 1-3, TYK2) were found to play a central role in cytokine receptor signalling (Ihle and Kerr, 1995). Mutations in the membrane proximal cytoplasmic region that prevent JAK-binding and activation completely abolish the proliferative signalling capacities of, for example, GM-CSF-R and EPO-R (Miura et al, 1993; Quelle et al, 1994). Thus, activation of JAKs is essential for the mitogenic signalling function of HGF-Rs. Supporting this, it was found that overexpression of a kinase deficient form of JAK2 interfered with EPO-induced proliferative responses (Zhuang et al, 1994). Upon activation, JAKs Tyr phosphorylate STAT (signal transducer and activator of transcription) proteins. The Tyrphosphorylated STAT proteins form complexes that translocate directly to 
the nucleus, where they bind to specific transcription regulatory sequences in the genomic DNA and induce gene expression. So far, six distinct STAT proteins, plus a number of isoforms, have been identified in mammalian cells. Because HGF-Rs show specificity in their ability to recruit and activate STATs, it was suggested that diversity within the type of STAT complexes activated might contribute to the nature of the diverse cellular responses to a given HGF (Ihle and Kerr, 1995). Evidence in support of this is provided in recent in vivo and in vitro studies in which gene knock-out and dominant negative mutant strategies were applied to specifically block the expression and/or function of STATs (Mui et al, 1995; Durbin et al, 1996; Kaplan et al, 1996; Meraz et al, 1996; Shimoda et al, 1996; Takeda et al, 1996; Thierfelder et al, 1996). Given the central role of JAKs and STATs in driving cellular responses to various HGFs and cytokines, it may be suggested that aberrations in the function of JAKs or STAT function would have profound clinical effects.

The $\mathrm{p} 21^{\mathrm{Ras}} / \mathrm{MAPkinase}$ pathway is a second major route implicated in HGF-R signalling. This route is more complex than the JAK/STAT pathway and is activated via a variety of intermediate signalling complexes. Adaptor proteins acting upstream of $\mathrm{p} 21^{\text {Ras }}$ include Shc, Grb2 and the protein tyrosine phosphatase SHP-2. Shc and Grb2, or SHP-2 and Grb2, form complexes with the nucleotide exchange factor Sos (Son of sevenless), which results in the activation of $\mathrm{p} 21^{\mathrm{Ras}}$. The $\mathrm{p} 21^{\mathrm{Ras}}$ pathway is involved in mediating HGF supported survival of haemopoietic cells (Kinoshita et al, 1995). Furthermore, signals given via this route have been found to cooperate with JAK/STAT signals in controlling cell cycle progression. The cooperation between the JAK/STAT and $\mathrm{p} 21^{\text {Ras }}$ pathways is more intimate than was initially thought. Activation of the Ras route by, for example, G-CSF-R, cannot be achieved independent of JAKs (Barge et al, 1996). This is because JAKs are (directly or indirectly) responsible for the phosphorylation of Tyr residues in the receptor molecules, which serve as binding sites for signalling substrates implicated in the $\mathrm{p} 21^{\text {Ras }}$ route. The opposite is also true that, although phosphorylation on Tyr residues by JAKs suffices for STAT complex formation and DNA binding, it appears that subsequent induction of transcription by STAT complexes requires an additional phosphorylation event, on serine(Ser) residues (Wen et al, 1995). This is achieved via members of the MAPK family, some of which are activated via $\mathrm{p} 21^{\text {Ras }}$ (David et al, 1995).

\section{Class I cytokine receptor defects in haemopoietic diseases}

Given the strict dependence of haemopoietic progenitor cells on HGFs for proliferation, survival and differentiation in vitro, it had been anticipated that mutations in HGF-R genes could have a role in the development of certain haemopoietic disorders. Initial evidence in support of this came from the demonstration that a truncated form of the TPO-R, previously referred to as MPL, that lacks the major part of the extracellular domain, is transduced by murine myeloproliferative leukaemia virus (MPLV) and immortalizes haemopoietic progenitor cells (Souyri et al, 1990). In 
addition, it was shown that alterations in the extracellular domain of EPO$\mathrm{R}$ and the common $\beta$ chain of IL-3R/IL-5R/GM-CSF-R, which confer ligand independent activation of these receptors, contributed to the development of erythroid and myeloid leukaemias in mice, respectively (Longmore and Lodish, 1991; Jenkins et al, 1995). However, transforming mutations in the $\beta_{c}$ receptor chain have so far not been detected in human myeloid leukaemia (Freeburn et al, 1996). Mutations in growth factor receptor genes affecting the signalling function of the receptor proteins have been found in four clinical haematological conditions: X-linked severe combined immunodeficiency (X-linked SCID), benign erythrocytosis, SCN and AML. The disease locus in X-linked SCID is positioned at $X q 13.1$ and comprises the gene encoding the common chain $\left(\gamma_{c}\right)$ of the IL-2, IL-7, IL-15 and possibly the IL-4 and IL-13 receptor complexes (Noguchi et al, 1993; Puck et al, 1993). X-linked SCID patients express mutations in the $\gamma_{c}$ gene. These mutations are not limited to a single critical region, but occur at multiple positions in the gene. They may lead to protein truncations or structural alterations in the extracellular domain, giving rise to non-ligand binding or soluble forms of the $\gamma_{c}$ protein (Noguchi et al, 1993; Puck et al, 1993; Voss et al, 1994; Pepper et al, 1995). Alternatively, a portion of the cytoplasmic domain is truncated, which contains the cytoplasmic SH2 domain of the $\left(\gamma_{c}\right)$ receptor sub-unit that can predictively bind to downstream, as yet unidentified, signalling molecules, as well as the binding site of the PTK JAK3 (Johnston et al, 1994, Pepper et al, 1995). Two sets of observations have established that the inability to activate JAK3 specifically determines the disease phenotype in X-linked SCID. First, double knock-out ('null') mice lacking $J A K 3$ genes display a SCID phenotype that is indistinguishable from that of $\gamma_{\mathrm{c}}$ null mice (Disanto et al, 1995; Nosaka et al, 1995). Second, a number of cases of autosomal SCID patients have recently been reported with inactivating mutations in the $J A K 3$ gene. These patients display clinical symptoms similar to $\mathrm{X}$-linked SCID (Macchi et al, 1995; Russell et al, 1995).

Benign erythrocytosis is a dominant autosomal condition characterized by a mild increase in red blood cell counts with normal serum levels of EPO. The condition has been associated with mutations in the EPO-R. So far, two pedigrees with distinct types of mutations have been identified. One is a nonsense mutation that results in the deletion of 70 carboxyterminal amino acids (De la Chapelle et al, 1993). The second is an insertion resulting in a frameshift and a premature stop codon, causing a deletion of 64 carboxy-terminal amino acids (Sokol et al, 1995). As a result of these truncations, the binding site for the protein tyrosine phosphatase SHP-1, at Tyr 449 of EPO-R, is lost (Klingmüller et al, 1995). Because SHP-1 is a negative regulator of JAK2 activation by EPO, the mitogenic response to EPO is enhanced as a result of these truncations. This provides a plausible mechanistical explanation for the increased red blood cell production in these individuals (Youssoufian et al, 1993), which is further supported by the observation that SHP-1 deficient $\left(m e^{v} / m e^{v}\right)$ mice also demonstrate an increased sensitivity to EPO (Van Zant and Schultz, 1989). 


\section{G-CSF receptor defects in SCN and AML}

A maturation arrest of granulocytic progenitor cells in the bone marrow leads to profound absolute neutropenia in SCN. As a result, patients suffer from severe and life threatening opportunistic infections. SCN diagnosis is usually made early in infancy and the disease has a variable inheritance. Although SCN was originally described, in Swedish families, as an autosomal recessive disorder (Kostmann, 1956), autosomal dominant forms of SCN have also been identified. Additionally, SCN cases without a family history have been reported. This variability in genetic background implicates that SCN is a heterogeneous disease condition in which defects in different genes may give rise to a largely similar disease phenotype.

Because GM-CSF and G-CSF are major growth factors involved in the production of neutrophilic granulocytes, the possibility that SCN patients might clinically benefit from treatment with these growth factors has received major interest. Initial studies in which $\mathrm{SCN}$ patients were treated with GM-CSF showed that myelopoiesis was enhanced as a result of the treatment, leading to a significant rise in absolute leukocyte counts. However, with few exceptions, GM-CSF led to increased eosinophil and monocyte counts, whereas neutrophil levels remained low (Vadhan-Raj et al, 1990; Welte et al, 1990). These clinical observations indicated that GMCSF treatment of SCN patients is generally not sufficient to restore the intrinsic defect in neutrophilic maturation. In contrast, G-CSF treatment of SCN patients leads to large increases in functional neutrophils, resulting in significant clinical improvement (Bonilla et al, 1989; Welte et al, 1990). Therefore, it was hypothesized that reduced levels of biologically active G-CSF might be involved in the pathogenesis of SCN. However, production of G-CSF by mononuclear cells and bone marrow stroma cells from SCN patients appeared to be normal and G-CSF serum levels often higher than normal, which excluded defective G-CSF production as a possible cause of neutropenia in these patients (Mempel et al, 1991; Pietsch et al, 1991; Guba et al, 1994).

A randomized, controlled phase III trial showed that $90 \%$ of SCN patients respond to G-CSF treatment by increases in their absolute neutrophil counts, although generally to levels which are still below normal (Dale et al, 1993). This resulted in approximately $50 \%$ reduction in the incidence and duration of infection-related events and almost $70 \%$ reduction in duration of antibiotic use. In patients in which G-CSF therapy does not result in sustained and sufficiently increased numbers of circulating neutrophils, infection-related mortality remains a major concern (Ryan et al, 1995; Zeidler et al, 1996).

A second major clinical complication is that SCN patients have an increased probability of developing myelodysplasia (MDS) and/or AML with poor prognosis (Gillio and Gabrilove, 1993). The incidence of progression to leukaemia among SCN patients registered by the Severe Congenital Neutropenia International Registry (SCN-IR) is 16 out of 220 or $7.27 \%$, as reported in September 1996 (Freedman et al, 1996). The follow-up of this cohort of patients, who all received G-CSF therapy, is still 
relatively short ( 8 years or less). It is therefore possible that this figure is still an underestimation of the frequency of AML progression.

The variability in clinical responses of SCN patients to G-CSF is a further illustration of the heterogeneous nature of $\mathrm{SCN}$. The fact that most patients respond to pharmacological but not to normal dosages of G-CSF raised the suggestion that abnormalities in the expression or growth factor binding abilities of G-CSF-R could play a role in SCN. However, the observation that neutrophils from SCN patients expressed normal levels of G-CSF-R with normal G-CSF binding affinity argued against this idea (Kyas et al, 1992). An alternative explanation is that the cytoplasmic signalling function of G-CSF-R is affected in SCN. Support for this possibility came from the identification of an $S C N$ patient with a somatic mutation in the G-CSF-R gene (Dong et al, 1994). This mutation resulted in the truncation of the C-terminal cytoplasmic region of the G-CSF-R that is critical for its maturation signalling function (Dong et al, 1993; Fukunaga et al, 1993). The mutation was heterozygous and present in the myeloid cells of the patient, but not in other haemopoietic lineages (erythroid, $T$ and B lymphocytic) or skin fibroblasts from either the patient or his parents, indicating that it had occurred in a progenitor cell committed to the myeloid lineage. Besides lacking maturation signalling abilities, the truncated G-CSF-R protein also misses a C-terminal element that negatively regulates proliferation (Dong et al, 1994). Experiments in which wildtype and mutant $G-C S F-R$ were co-expressed at comparable levels in 32D cells showed that maturation signalling from the wildtype form is inhibited by the truncated G-CSF-R form in a dominant negative fashion (Dong et al, 1995a). This, in combination with the fact that the affected myeloid progenitor cells had clonally expanded in the patient, suggested that expression of the mutant $G-C S F-R$ induced or contributed to a premalignant status of the cells. In further support of this, the leukaemic cells from two AML patients with a history of $\mathrm{SCN}$ also had mutations in G-CSF-R, again truncating the $\mathrm{C}$-terminal maturation-inducing region (Dong et al, 1995a). Among 20 other cases, two additional patients with $G-C S F-R$ mutations in the critical region were identified, both of which developed AML. Notably, $G-C S F-R$ mutations were not detected in SCN patients from the original pedigree of Kostmann, i.e. the autosomal recessive variant (Dong et al, 1997).

Tidow et al (1997) recently reported the presence of $G-C S F-R$ nonsense mutations in four out of $28 \mathrm{SCN}$ patients. One of these (patient FR) had already been included in the analysis of Dong et al (1997), but the others represented novel cases. Two of their four patients with $G \cdot C S F-R$ mutations, including FR, developed AML. Thus, from a total of 50 , seven patients (14\%) had mutations in the $G-C S F-R$ gene, four of whom showed disease progression to AML. Importantly, a $G-C S F-R$ mutation was detected after leukaemic progression but not at the neutropenic stage of the disease. This patient suffered from an inherited recessive form of SCN, with a brother also being severely neutropenic. Neither this affected brother nor the healthy parents had a $G-C S F-R$ mutation. These observations provided further support for the notion that a $G-C S F-R$ mutation truncating 
the C-terminal domain is associated with leukaemic progression in $\mathrm{SCN}$, but also suggested that this defect had not been the primary cause of the neutropenia, at least in this familial form of SCN.

To establish to what extent G-CSF-R mutations contribute to the disease phenotype, experiments in which these mutations are targeted into the haemopoietic cells of mice and the consequences studied in vivo are warranted. So far, it has been demonstrated that $G-C S F$ or $G-C S F-R$ null mice suffer from chronic neutropenia, further establishing the essential role of G-CSF in neutrophil development (Lieschke et al, 1994; Liu et al, 1996). Strikingly, the neutropenia in these knock-out mice is not as deep as in SCN patients. Apparently, other growth factors can to a certain extent substitute for G-CSF, as observed by Liu et al. in their $G-C S F-R$ null model (Liu et al, 1996). The defects in SCN may be more severe because they affect the maturation abilities in such a way that compensatory mechanisms are ineffective. In the context of the $G-C S F-R$ mutations, this could be because truncated G-CSF-R combine defective maturation with enhanced proliferation signalling (Dong et al, 1993). These gross disturbances of the proliferation/maturation balance and changes in cell cycle kinetics in response to G-CSF could make the compensatory mechanisms ineffective, which would explain the accumulation of immature cells in the bone marrow that is characteristically observed in $\mathrm{SCN}$ patients.

The majority of SCN patients do not express $G-C S F-R$ mutations. By analogy to the severe combined immunodeficiency syndrome, in which mutations in either the $\gamma_{c}$ or Jak3 genes give rise to the same clinical symptoms, it is tempting to speculate that these patients could have defects in signalling substrates directly downstream of the G-CSF-R. The signalling properties of G-CSF-R on bone marrow and blood cells of SCN patients have been investigated to a limited extent. For instance, it was shown that G-CSF-R, are not hampered in their ability to activate the JAK2 kinase (Rauprich et al, 1995). Taking into account the localization of the $G-C S F-R$ mutations, this is not surprising because activation of JAK2 is mediated via the membrane proximal cytoplasmic region of G-CSF-R. Rather, potential defects in the signalling function downstream of G-CSF$\mathrm{R}$ might be expected to involve substrates activated via the carboxyterminal region of the receptor.

A STAT family member that is prominently activated by G-CSF is STAT3 (De Koning et al, 1996). Although little is known of the target genes of activated STAT3 complexes that are implicated in neutrophilic maturation, recent work has indicated that STAT3 is involved in IL-6induced macrophage differentiation (Nakajima et al, 1996). G-CSFinduced activation of STAT3 depends on the recruitment of STAT3 to the G-CSF-R via binding of STAT3-SH2 domains to multiple phosphotyrosines of the activated G-CSF-R. Three of these Tyrs are located in the C-terminal region associated with maturation signalling, raising the possibility that STAT3 could be involved in the regulation of neutrophilic development. However, truncated G-CSF-R forms from SCN patients are still able to activate STAT3, indicating that the lack of maturation signalling function of the mutant receptors cannot simply be attributed to 
disturbed STAT3 activation. On the other hand, the truncated G-CSF-R forms have lost their ability to activate the Shc protein, involved in the $\mathrm{p} 21^{\text {Ras }}$ pathway. Recently it has been demonstrated that Shc activation is required for differentiation induction via the TPO-R (Alexander et al, 1996). In contrast, studies with Tyr substitution mutants of G-CSF-R indicated that neutrophilic differentiation induced by G-CSF does not depend on Shc activation (De Koning et al, 1997). In fact, it is still unclear which signalling substrates are critical for G-CSF-R-induced neutrophilic maturation.

Mutations affecting the function of the $G-C S F-R$ can also be found in AML patients without a disease history of congenital neutropenia. Thus far, two such cases have been reported. One patient expressed a mutation truncating the C-terminal maturation domain, similar to those detected in SCN (Carpeti et al, 1997). In the second patient, AML blasts showed high expression of a new $G-C S F-R$ splice variant termed SD, in which the carboxy-terminus is altered due to a change in the reading frame (Dong et al, 1995b). Analysis of cDNA and corresponding genomic sequences revealed a $\mathrm{G}$ to $\mathrm{A}$ transition next to a (cryptic) splice donor site involved in the alternative RNA splicing. The SD splice variant was unable to transduce proliferation and maturation signals upon transfer to murine cell systems. In agreement with this, the primary AML blast cells of this patient failed to respond to G-CSF in proliferation assays in vitro, whereas the responsiveness to IL-3 or GM-CSF was maintained. These observations implicate that G-CSF-R dysfunction, either caused by structural defects in the G-CSF-R (rarely) or by abnormalities in downstream signalling components mediating maturation signalling, can play a role in the pathogenesis of de novo AML.

\section{Acknowledgements}

This work was supported by the Dutch Cancer Society and the Netherlands Organization for Scientific Research (NWO).

\section{REFERENCES}

Alexander WS, Maurer AB, Novak U \& Harrison-Smith M (1996) Tyrosine-599 of the c Mpl receptor is required for She phosphorylation and the induction of cellular differentiation. EMBO Journal 15: $6531-6540$.

Barge RMY, de Koning JP \& Pouwels K et al (1996) Tryptophan 650 of human granulocyte colonystimulating factor (G-CSF) receptor, implicated in the activation of JAK2, is also required for G-CSF-mediated activation of signaling complexes of the p21Ras route. Blood 87: 21482153.

Bazan JF (1990) Structural design and molecular evolution of a cytokine receptor superfamily. Proceedings of the National Academy of Sciences of the USA 87: 6934-6938.

*Bonilla MA, Gillio AP, Ruggeiro M et al (1989) Effects of recombinant human granulocyte colonystimulating factor on neutropenia in patients with congenital agranulocytosis. New England Journal of Medicine 320: 1574-1580.

Carapeti M, Soede-Bobok AA, Hochhaus A et al (1997) A dominant negative mutation of the G-CSF receptor truncating the $\mathrm{C}$-terminal maturation domain in a patient with acute myeloid leukemia without congenital neutropenia. Leukemia 11: 1005-1008. 
De la Chapelle A, Träskelin AL \& Juvonen E (1993) Truncated erythropoietin receptor causes dominantly inherited benign human erythrocytosis. Proceedings of the National Academy of Sciences of the USA 90: 4495-4499.

Cosman D (1993) The hematopoietin receptor superfamily. Cytokine 5: 95-106.

*Dale DC, Bonilla MA, Davis MW et al (1993) A randomized controlled phase III trial of recombinant human granulocyte colony-stimulating factor (Filgrastim) for treatment of severe chronic neutropenia. Blood 81: 2496-2502.

David M, Petricoin III E, Benjamin C et al (1995) Requirement for MAP kinase (ERK2) activity in interferon $\alpha$ - and interferon $\beta$-stimulated gene expression through STAT proteins. Science 269: $1721-1723$.

Disanto JP, Muller W, Guy-Grand D et al (1995) Lymphoid development in mice with a targeted deletion of the interleukin-2 receptor $\gamma \mathrm{c}$ chain. Proceedings of the National Academy of Sciences of the USA 92: $377-381$.

* Dong F, Van Buitenen C, Pouwels K et al (1993) Distinct cytoplasmic regions of the granulocyte colony-stimulating factor receptor involved in induction of proliferation and maturation. Molecular and Cellular Biology 13:7774-7781.

*Dong F, Hoefsloot LH, Schelen AM et al (1994) Identification of a nonsense mutation in the granulocyte colony-stimulating factor receptor in severe congenital neutropenia. Proceedings of the National Academy of Sciences of the USA 91: 4480-4484.

*Dong F, Brynes RK, Tidow $N$ et al (1995a) Mutations truncating the C-terminal maturation region of the G-CSF receptor in acute myeloid leukemia preceded by severe congenital neutropenia. New England Journal of Medicine 333: 487-493.

Dong $F$, Van Paassen M, Van Buitenen $C$ et al (1995b) A point mutation in the granulocyte colonystimulating factor receptor (G-CSF-R) gene in a case of acute myeloid leukemia results in the overexpression of a novel G-CSF-R isoform. Blood 85: 902-912.

Dong F, Dale DC, Bonilla MA et al (1997) Mutations in the granulocyte colony-stimulating factor gene in patients with severe congenital neutropenia. Leukemia 11: 120-125.

Durbin JE, Hackenmiller R, Simon MC \& Levy DE (1996) Targeted disruption of the mouse Stat I gene results in compromised innate immunity to viral disease. Cell 85: 443-450.

Freeburn RW, Gale RE, Wagner HM \& Linch DC (1996) The beta subunit common to the GM-CSF, IL-3 and IL-5 receptors is highly polymorphic but pathogenic point mutations in patients with acute myeloid leukaemia (AML) are rare. Leukemia 10: 123-129.

Freedman MH, Bonilla MA, Boxer $L$ et al (1996) MDS/AML in patients with severe chronic neutropenia (SCN) receiving G-CSF. Blood 88 (supplement 1): $\mathrm{S} 448$.

*Fukunaga R, Ishizaki-Ikeda E \& Nagata S (1993) Growth and differentiation signals mediated by different regions in the cytoplasmic domain of granulocyte colony-stimulating factor receptor. Cell 74: $1079-1087$.

Fukunaga R, Ishizaka-Ikeda E, Pan C-X et al (1991) Functional domains of the granulocyte colonystimulating factor receptor. EMBO Journal 10: 2855-2865.

Gillio AP \& Gabrilove IL (1993) Cytokine treatment of inherited bone marrow failure syndromes. Blood 81: $1669-1674$.

Guba SC, Sartor CA, Hutchinson R et al (1994) Granulocyte colony-stimulating factor (G-CSF) production and G-CSF receptor structure in patients with congenital neutropenia. Blood 83: 1486-1492.

Ihle JN \& Kerr IM (1995) Jaks and Stats in signaling by the cytokine receptor superfamily. Trends in Genetics 11: 69-74.

Jenkins BJ, D'Andrea R \& Gonda TJ (1995) Activating point mutations in the common $\beta$ subunit of the human GM-CSF, IL-3 and $I L-5$ receptors suggest the involvement of $\beta$ subunit dimerization and cell type-specific molecules in signalling. EMBO Journal 14: 4276-4287.

Johnston JA, Kawamura M, Kirken RA et al (1994) Phosphorylation and activation of the Jak-3 kinase in response to interleukin-2. Nature 370: 151-153.

Kaplan MH, Sun X-L, Hoey $T$ \& Grusby MJ (1996) Impaired IL-12 responses and enhanced development of Th cells in Stat-4-deficient mice. Nature 382: 174-177.

Kinoshita T, Yokota T, Arai K-I \& Miyajima A (1995) Suppression of apoptotic death in hematopoietic cells by signalling through the IL-3/GM-CSF receptors. EMBO Journal 14: $266-$ 275 .

* Klingmuiller U, Lorenz U, Cantley LC et al (1995) Specific recruitment of SH PTP 1 to the erythropoietin receptor causes inactivation of JAK2 and termination of proliferative signals. Cell 80 : $729-738$. 
De Koning JP, Dong F, Smith L et al (1996) The membrane-distal cytoplasmic region of human granulocyte colony-stimulating factor receptor is required for STAT3 but not STAT 1 homodimer formation. Blood 87: 1335-1342.

De Koning JP, Smith L, Schelen AM et al (1997) Distinct mechanisms of p21Ras activation by GCSF receptor: implications for G-CSF mediated regulation of proliferation and neutrophilic maturation. Blood 88 (supplement 1): S446.

Kostmann R (1956) Infantile genetic agranulocytosis. Acta Paediatrica Scandinavia, 45 (supplement 105): $\mathrm{S} 1-\mathrm{S} 78$.

Kyas U, Pietsch T \& Welte K (1992) Expression of receptors for granulocyte colony-stimulating factor on neutrophils from patients with severe congenital neutropenia and cyclic neutropenia. Blood 79: 1144-1147.

*Lieschke GJ, Grail D, Hodgson G et al (1994) Mice lacking granulocyte colony-stimulating factor have chronic neutropenia, granulocyte and macrophage progenitor cell deficiency, and impaired neutrophil mobilization. Blood 84: 1737-1746.

*Liu F, Wu HY, Wesselschmidt R et al (1996) Impaired production and increased apoptosis of neutrophils in granulocyte colony-stimulating factor receptor deficient mice. Immunity 5: 491-501.

Longmore GD \& Lodish HF (1991) An activating mutation in the murine erythropoietin receptor induces erythroleukemia in mice: a cytokine receptor superfamily oncogene. Cell 1991: 1089-1102.

Macchi P, Villa A \& Gillani S (1995) Mutations of Jak-3 gene in patients with autosomal severe combined immunodeficiency (SCID). Nature 377: 65-68.

Mempel K, Pietsch T, Menzel T et al (1991) Increased serum levels of granulocyte colony-stimulating factor in patients with severe congenital neutropenia. Blood 77: 1919-1922.

Meraz MA, White JM, Sheehan KCF et al (1996) Targeted disruption of the Stat l gene in mice reveals unexpected physiologic specificity in the JAK-STAT signaling pathway. Cell $84: 431-442$.

Miura O, Cleveland JL \& thle JN (1993) Inactivation of erythropoietin receptor function by point mutations in a region having homology with other cytokine receptors. Molecular and Cellular Biology 13: 1788-1795.

Miyajima A, Mui ALF, Ogorochi T \& Sakamaki K (1993) Receptors for granulocyte-macrophage colony-stimulating factor, interleukin-3, and interleukin-5. Blood 82: 1960-1974.

Mui ALF, Wakao H, O'Farrell AM et al (1995) Interleukin-3, granulocyte-macrophage colonystimulating factor and interleukin-5 transduce signals through two STAT5 homologs. EMBO Journal 14: 1166-1175.

Nakajima K, Yamanaka Y, Nakae K et al (1996) A central role for Stat3 in IL-6-induced regulation of growth and differentiation in M1 leukemia cells. EMBO Journal 15: 3651-3658.

* Noguchi M, Yi H, Rosenblatt HM et al (1993) Interleukin-2 receptor $\gamma$ chain mutation results in Xlinked severe combined immunodeficiency in humans. Cell 73: 147-157.

Nosaka T, Van Deursen JM \& Tripp RA (1995) Defective lymphoid development in mice lacking JAK3, Science 270: 800-802.

Pepper AE, Buckley RH, Small TN \& Puck JM (1995) Two mutational hotspots in the interleukin-2 receptor gamma chain gene causing human X-linked severe combined immunodeficiency. American Journal of Human Genetics 57: 564-571.

Pietsch T, Bührer C, Mempel $\mathrm{K}$ et al (1991) Blood mononuclear cells from patients with severe congenital neutropenia are capable of producing granulocyte colony-stimulating factor. Blood 77: $1234-1237$.

Porteu F, Rouyez MC, Cocault $\mathrm{L}$ et al (1996) Functional regions of the mouse thrombopoietin receptor cytoplasmic domain: evidence for a critical region which is involved in differentiation and can be complemented by erythropoietin. Molecular and Cellular Biology 16: 2473-2482.

Puck JM, Deschenes SM, Porter JC et al (1993) The interleukin-2 receptor gamma chain maps to Xq13.1 and is mutated in severe combined immunodeficiency, SCIDX1. Human Molecular Genetics 2: 1099-1104.

Quelle FW, Sato N \& Witthuhn BA (1994) Jak2 associates with the $\beta_{c}$ chain of the receptor for granulocyte-macrophage colony-stimulating factor, and its activation requires the membraneproximal region. Molecular and Cellular Biology 14: 4335-4341.

Rauprich P, Kasper B, Tidow N \& Welte K (1995) The protein tyrosine kinase JAK2 is activated in neutrophils from patients with severe congenital neutropenia. Blood 86: 4500-4505.

Russell SM, Tayebi N, Nakajima $\mathrm{H}$ et al (1995) Mutation of JAK3 in a patient with SCID: essential role of JAK3 in lymphoid development. Science 270: 797-800. 
Ryan M, Will AM, Testa N et al (1995) Severe congenital neutropenia unresponsive to G-CSF. British Journal of Haematology 91: 43-45.

Shimoda K, van Deursen J, Sangster MY et al (1996) Lack of IL-4-induced Th2 response and IgE class switching in mice with disrupted Stat6 gene. Nature 380: 630-633.

Sokol L, Luhovy M, Guan Y et al (1995) Primary familial polycythemia: a frameshift mutation in the erythropoietin receptor gene and increased sensitivity of erythroid progenitors to erythropoietin. Blood 86: 15-22.

Souyri M, Vigon I, Penciolelli J-F et al (1990) A putative truncated cytokine receptor gene transduced by the myeloproliferative leukemia virus immortalizes hematopoietic progenitors. Cell 63: $1137-1147$.

Takeda K, Tanaka T, Shi $W$ et al (1996) Essential role of Stat6 in IL-4 signalling. Nature 380: $627-630$

Thierfelder WE, van Deursen JM, Yamamoto K et al (1996) Requirement for Stat4 in interleukin-12 mediated responses of natural killer cells. Nature 382: 171-174

Tidow N, Pilz C, Teichmann B et al (1997) Clinical relevance of point mutations in the granulocyte colony-stimulating factor receptor gene in patients with severe congenital neutropenia. Blood 89: 2369-2375.

Vadhan-Raj S, Jeha SS, Buescher S et al (1990) Stimulation of myelopoiesis in a patient with congenital neutropenia: biology and nature of response to recombinant human granulocytemacrophage colony-stimulating factor. Blood 75: 858-864.

Voss SD, Hong R \& Sondel PM (1994) Severe combined immunodeficiency, interleukin-2 (IL-2), and the IL-2 receptor: experiments of nature continue to point the way. Blood 83: 626-635.

Welte K, Zeidler C, Reiter A et al (1990) Differential effects of granulocyte-macrophage colonystimulating factor and granulocyte colony-stimulating factor in children with severe congenital neutropenia. Blood 75: 1056-1063.

Wen Z, Zhong Z \& Darnell JE (1995) Maximal activation of transcription by Stat 1 and Stat3 requires both tyrosine and serine phosphorylation. Cell 82: 241-250.

Yamanaka Y, Nakajima K, Fukada $T$ et al (1996) Differentiation and growth artest signals are generated through the cytoplasmic region of gp 130 that is essential for Stat3 activation. EMBO Journal 15: $1557-1565$.

Youssoufian H, Longmore G, Neumann D et al (1993) Structure, function and activation of the Epo receptor. Blood 81: 2223-2236.

Van Zant G \& Schultz L (1989) Hematologic abnormalities of the immunodeficient mouse mutant, viable motheaten (mev). Experimental Hematology 17: 81-87.

Zeidler C, Bonilla MA, Boxer $L$ et al (1996) Report on patients with severe chronic neutropenia (SCN) refractory to G-CSF. Blood 88 (supplement 1 ): \$349.

Zhuang H, Patel SV, He T et al (1994) Inhibition of erythropoietin-induced mitogenesis by a kinasedeficient form of Jak2. Joumal of Biological Chemistry 269: 21411-21414. 